\title{
Evaluation of pancreatic cancer cell migration with multiple parameters in vitro by using an optical real-time cell mobility assay device
}

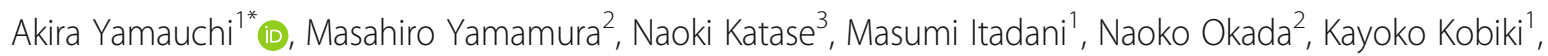
Masafumi Nakamura ${ }^{4}$, Yoshiyuki Yamaguchi ${ }^{2}$ and Futoshi Kuribayashi ${ }^{1}$

\begin{abstract}
Background: Migration of cancer cell correlates with distant metastasis and local invasion, which are good targets for cancer treatment. An optically accessible device "TAXIScan" was developed, which provides considerably more information regarding the cellular dynamics and less quantity of samples than do the existing methods. Here, we report the establishment of a system to analyze the nature of pancreatic cancer cells using TAXIScan and we evaluated lysophosphatidic acid (LPA)-elicited pancreatic cell migration.

Methods: Pancreatic cancer cell lines, BxPC3, PANC-1, AsPC1, and MIAPaCa-2, were analyzed for adhesion as well as migration towards LPA by TAXIScan using parameters such as velocity and directionality or for the number of migrated cells by the Boyden chamber methods. To confirm that the migration was initiated by LPA, the expression of LPA receptors and activation of intracellular signal transductions were examined by quantitative reverse transcriptase polymerase reaction and western blotting.

Results: Scaffold coating was necessary for the adhesion of pancreatic cancer cells, and collagen I and Matrigel were found to be good scaffolds. BxPC3 and PANC-1 cells clearly migrated towards the concentration gradient formed by injecting $1 \mu \mathrm{L}$ LPA, which was abrogated by pre-treatment with LPA inhibitor, Ki16425 (IC 50 for the directionality $\approx 1$. $86 \mu \mathrm{M})$. The LPA dependent migration was further confirmed by mRNA and protein expression of LPA receptors as well as phosphorylation of signaling molecules. $L P A_{1}$ mRNA was highest among the 6 receptors, and $L P A_{1}, L P A_{2}$ and $L P A_{3}$ proteins were detected in BxPC3 and PANC-1 cells. Phosphorylation of Akt (Thr308 and Ser473) and p42/44MAPK in BXPC3 and PANC-1 cells was observed after LPA stimulation, which was clearly inhibited by pre-treatment with a compound Ki16425.

Conclusions: We established a novel pancreatic cancer cell migration assay system using TAXIScan. This assay device provides multiple information on migrating cells simultaneously, such as their morphology, directionality, and velocity, with a small volume of sample and can be a powerful tool for analyzing the nature of cancer cells and for identifying new factors that affect cell functions.
\end{abstract}

Keywords: Migration, Chemotaxis, Lipid mediator, Inhibitor, TAXIScan, Metastasis

\footnotetext{
* Correspondence: akiray@med.kawasaki-m.ac.jp

'Department of Biochemistry, Kawasaki Medical School, 577 Matsushima,

Kurashiki, Okayama 701-0192, Japan

Full list of author information is available at the end of the article
}

(c) The Author(s). 2017 Open Access This article is distributed under the terms of the Creative Commons Attribution 4.0 International License (http://creativecommons.org/licenses/by/4.0/), which permits unrestricted use, distribution, and reproduction in any medium, provided you give appropriate credit to the original author(s) and the source, provide a link to the Creative Commons license, and indicate if changes were made. The Creative Commons Public Domain Dedication waiver (http://creativecommons.org/publicdomain/zero/1.0/) applies to the data made available in this article, unless otherwise stated. 


\section{Background}

Migration of cancer cells correlates with distant metastasis and local invasion. This phenomenon involves various molecules including chemoattractants, trophic growth factors and their receptors, adhesion molecules, intracellular signaling molecules, motor proteins, and the cytoskeleton [1]. These molecules are orchestrated to help cells migrate to specific parts of the body or even spontaneously without an apparent destination. As cancer metastasis is directly associated with prognosis, controlling cancer cell migration is an effective strategy for treating the disease. Pancreatic cancer is among those with the poorest prognosis [2]. The treatment for this type of cancer is currently restricted as there are few effective drugs and knowledge regarding the nature of this cancer type is insufficient. New insights regarding this cancer and novel approaches for its treatment have long been awaited.

Lysophosphatidic acid (LPA) is a highly bioactive lipid mediator and is known to be involved in cancer cell migration, proliferation, and production of angiogenic factors [3]. In the process of cell migration, LPA works as a potent chemoattractant for various kinds of cells. Six receptors of LPA $\left(\mathrm{LPA}_{1}, \mathrm{LPA}_{2}, \mathrm{LPA}_{3}, \mathrm{LPA}_{4}, \mathrm{LPA}_{5}\right.$, and $\left.\mathrm{LPA}_{6}\right)$ are known and all of them are G-protein coupled [4-9]. Some cells express one of these receptors, while others express multiple receptors for LPA [10]. Several articles have reported that pancreatic cancer cell lines express LPA receptors and the cells migrate towards LPA, using Boyden chamber and/or Transwell culture methods, which involve counting the number of migrated cells [11-13].

TAXIScan is an assay device for studying cell dynamics in vitro and has been used in the analysis of both suspension (mostly hematopoietic) and adherent cells [14-22]. The device functions as an optically accessible system and provides two-dimensional images of cell migration. TAXIScan provides markedly more information including morphology as well as quantitative analysis compared to existing methods such as Boyden chamber method. This device consists of an etched silicon substrate and a flat glass plate, both of which form horizontal channels each with a micrometer-order depth and forms 2 compartments on either side of a channel. Cells are placed and aligned on one side, while a stimulating factor is injected to the other side (typically $1 \mu \mathrm{L}$ each of the cells and the stimulant). The cells react to the stable concentration gradient of the stimulant inside the horizontal channel [14]. The cell images are observed thereafter and filmed with a charge-coupled device camera located beneath the glass. By analyzing the cell images, many parameters can be determined including velocity, directionality, etc. [23-26].

The objective of this study is to establish TAXIScan as a system for pancreatic cancer research by using pancreatic cancer cell lines and to evaluate cancer cell migration in vitro for understanding the characteristics of this cancer cell type and for identifying new drugs to regulate cancer cell migration. Here, we show the adherence of cells to the scaffolds as well as LPA-elicited migration by TAXIScan, and by an existing method, the modified Boyden chamber method (Transwell). The LPA-elicited migration was confirmed by checking the expression of LPA receptors and the effect of an LPA inhibitor Ki16425.

\section{Methods \\ Reagents}

Fetal bovine serum (FBS) was obtained from Nichirei Biosciences Inc. (Tokyo, Japan); RPMI1640 and D-MEM were from Sigma-Aldrich (St. Louis, MO, USA); Collagen I, Matrigel (growth factor reduced), fibronectin, laminin, and collagen I pre-coated coverslips were obtained from Becton Dickinson (San Jose, CA, USA); fatty-acid-free bovine serum albumin (BSA) from Nacalai Tesque (Kyoto, Japan); LPA from Enzo Life Sciences Inc. (Farmingdale, NY, USA); Opti-MEM from Thermo Fisher Scientific Inc. (Waltham, MA, USA); Anti- LPA $1, \mathrm{LPA}_{3}, \mathrm{LPA}_{5}$, and $\mathrm{LPA}_{6}$ rabbit polyclonal antibodies from GeneTex Inc. (Irvine, CA, USA); anti-LPA 2 rabbit polyclonal antibody from Abgent (San Diego, CA, USA); and anti-LPA 4 rabbit polyclonal antibody from Acris Antibodies Inc. (San Diego, CA, USA); Ki16425 was purchased from Cayman Chemical (Ann Arbor, MI, USA).

\section{Maintenance of cells}

Human pancreatic cancer cell lines BxPC3 (ATCC CRL1687), PANC-1 (ATCC CRL-1469), and AsPC1 (ATCC CRL-1682) were obtained from the American Type Culture Collection (ATCC), and MIAPaCa-2 (RCB2094) and KATOIII (RCB2088) from Riken Cell Bank. PC3 and $211 \mathrm{H}$ were kindly provided by Dr. Masakiyo Sakaguchi. Cells were cultured and maintained in RPMI1640 with $10 \%$ FBS or in D-MEM with $10 \%$ FBS on $10-\mathrm{cm}$ diameter dishes as the standard procedure. Passaging of the cells was performed using PBS and Trypsin/EDTA solution when they were $80-90 \%$ confluent. All samples were handled according to the Declaration of Helsinki.

\section{Migration assay}

The Real-time cell mobility assay was performed by optical real-time cell mobility assay device "EZ-TAXIScan" (ECI, Inc., Kawasaki, Japan) as described previously [20], except for assembling the TAXIScan holder together with a coverslip pre-coated with the extracellular matrix. Briefly, coverslips were coated with collagen I $(100 \mu \mathrm{g} / \mathrm{mL})$, Matrigel (1/30 diluted solution with culture medium), fibronectin $(100 \mu \mathrm{g} / \mathrm{mL})$, laminin $(100 \mu \mathrm{g} / \mathrm{mL})$, or the culture medium, by incubating $100 \mu \mathrm{L}$ of each solution on a coverslip at room temperature for $1 \mathrm{~h}$ before assembling the TAXIScan holder. After collagen I was selected as the scaffold, collagen I pre-coated coverslips were used for the 
TAXIScan method. The pre-coated coverslip was washed once with $0.5 \mathrm{~mL}$ of PBS and was placed on the glass plate for TAXIScan. The TAXIScan holder was assembled according to the manufacturer's instructions. Cells were harvested by detaching from culture flasks using the same conditions as passaging. One $\mu \mathrm{L}$ of suspension prepared in the culture medium containing $2 \times 10^{6}$ cells $/ \mathrm{mL}$ was applied to the cell-injection side of TAXIScan holder and the cells (100 or less in most of the cases) were aligned at the edge of the micro-channel. After obtaining the first round of images, $1 \mu \mathrm{L}$ of the chemoattractant solution prepared in the chemotaxis buffer was added to the ligand-injection side of the device to initiate migration. The assay conditions were as follows: duration, $4 \mathrm{~h}$; interval, $5 \mathrm{~min}$; micro-channel depth, $10 \mu \mathrm{m}$; and temperature, $37^{\circ} \mathrm{C}$. Time-lapse images of cell migration were stored as electronic files on a computer hard disk and analyzed when needed. The morphologies of migrating cells were depicted by tracing the edge of cells and then superimposing the resulting outlines onto the initial image. Movies of the images were made and quantification of velocity and directionality was carried out through the "TAXIScan analyzer 2" software. The trajectory of each cell on the image was traced by clicking the center portion of each cell on the computer display. The velocity $(\mathrm{V})$ and the directionality (D) of each cell were calculated using the traced data as described previously [20,23]. The statistical analysis for the velocity and the directionality was done by the Kruskal-Wallis Test (Non-parametric ANOVA) followed by the Dunn's Multiple Comparisons Test, as the data did not show normal distribution in most cases [20].

The modified Boyden chamber method was performed using collagen I-coated polycarbonate membrane inserts ( $8 \mu \mathrm{m}$ pore size) in a 24 -well plate (CytoSelect $24-$ Well Cell Haptotaxis Assay kit, Cell Biolabs, Inc. San Diego, CA, USA) or Transwell Plate with non-coated polycarbonate membrane (Corning Incorporated, Corning, NY, USA), per the manufacturer's protocols. Briefly, the cells grown on a culture dish were detached with Trypsin/ EDTA solution, washed with PBS, and re-suspended in RPMI1640/HEPES buffer with $0.1 \%$ fatty-acid-free BSA (the chemotaxis buffer) to attain a density of $0.5 \times 10^{6}$ cells $/ \mathrm{mL}$. A total of $1.5 \times 10^{5}$ cells per well were placed in the upper chamber; the chemotaxis buffer with or without LPA was injected to the lower chamber, and then the plate was incubated at $37^{\circ} \mathrm{C}$ for $2 \mathrm{~h}$. The migrated cells were stained with the staining solution (supplied with the kit), observed under the microscope, and then lysed with the lysis solution (supplied with the kit) to quantify the number of migrated cells by measuring the absorbance at $560 \mathrm{~nm}$. The absorbance was calibrated with the numbers of cells by using the standard curve with a series of different cell numbers $(0,10,32,100,320$, 1000,3200 , and 10,000 cells).

\section{Quantitative reverse transcriptase polymerase reaction (qRT-PCR)}

Total RNA was extracted from the cells using the RNeasy kit (QIAGEN, Hilden, Germany). Cells were seeded on $10 \mathrm{~cm}$-diameter dishes until $80-90 \%$ confluency was attained. On the day of the experiment, the medium was removed, and the cells were washed with $5 \mathrm{~mL}$ PBS, followed by addition of lysis solution, per the manufacture's recommended procedure. Template DNA was prepared with extracted total RNA of each sample using Ready-To-Go You-Prime First-Strand Beads kit (GE Healthcare, Little Chalfont, UK) and $0.5 \mu \mathrm{L}$ each of 1st strand DNA per sample was used for quantitative polymerase reaction (qPCR) with Fast SYBR Green Master Mix reagent (Life Technologies, Carlsbad, CA, USA). Analysis was done after preparing samples in a 96-well plate; signal during PCR was detected by Step One Plus Realtime PCR system (Life Technologies). The primers used are given in Additional file 1: Table S1. $\beta$-actin was used as an internal control for normalization of data. Data were analyzed by the software accompanied with the PCR system.

\section{Protein expression and phosphorylation detection}

Cells were seeded on 10-cm-diameter dishes until 80-90\% confluency was attained. On the day of the experiment, cells were rinsed once with $5 \mathrm{~mL}$ of serum free OptiMEM and then stimulated with $1 \mu \mathrm{M}$ LPA prepared in the chemotaxis assay buffer (0.1\%BSA in RPMI1640) prewarmed at $37{ }^{\circ} \mathrm{C}$ for $30 \mathrm{~s}, 2 \mathrm{~min}$, or $5 \mathrm{~min}$. Immediately after stimulation, the medium was replaced with ice-cold chemotaxis assay buffer and cells were kept on ice until lysis was done. Cells were lysed with ice-cold lysis buffer from the PathScan RTK Signaling Antibody Array kit (Cell Signaling Technology, Danvers, MA, USA) per the manufacture's procedure. Cell lysate was kept at $-70{ }^{\circ} \mathrm{C}$ until the PathScan phosphorylation array or SDS-PAGE/ western blotting was performed. For western blotting, each cell lysate was subjected to SDS-PAGE, blotting, and antibody reaction. The pre-stained protein marker (Bio-Rad, Hercules, CA, USA) or the CruzMarker protein marker (Santa Cruz Biotechnology, Santa Cruz, CA, USA) was used to estimate the molecular weight of probed bands. Protein bands were visualized with ECL prime (GE Healthcare) and detected by LAS-4000 mini device (GE Healthcare). The list of the phosphorylated proteins for the array is shown in Additional file 2: Table S2.

\section{Results}

Establishing the optical real-time migration assay system for pancreatic cancer cells

We established the assay system for pancreatic cells using optically accessible horizontal cell mobility assay device, EZ-TAXIScan. This device has been used for 
monitoring chemotaxis assays mostly for hematopoietic cells such as neutrophils, monocytes/macrophages, dendritic cells, eosinophils, and lymphocytes [14-25]. In the case of adherent cells, like the cancer cells, additional procedures may be required for retrieving the optimal response from cells, such as scaffold coating [26]. Therefore, we compared different coatings on glass for facilitating pancreatic cell migration. Human collagen I, fibronectin, laminin, and Matrigel (growth factor reduced) were examined as scaffold substances coated on the glass plate inside the TAXIScan chamber. Among these materials, collagen I and Matrigel showed good performances (Fig. 1) (An additional movie file shows this in more detail [see Additional file 3]). Without coating, the cells did not attach well onto the glass plate (Fig. 1a) and did not show good migration (Fig. 1b). On the glass coated with collagen I or Matrigel, most cells attached and spread well even without a stimulant such as the chemoattractant (Fig. 1a). On the glass coated with collagen I or Matrigel, BxPC3 cells migrated towards LPA (Fig. 1b).

LPA is known as a chemoattractant for cancer cells. To observe chemotactic migration of the pancreatic cancer cells towards LPA using the TAXIScan system, we used different concentrations of LPA to seek an optimal concentration for migration and observed that $1 \mu \mathrm{M}$ of LPA was optimal for BxPC3 and PANC-1 cells (Fig. 2a) (An additional movie file shows this in more detail [see Additional file 4]). In the case of AsPC1 and MIAPaCa-2 cells, very few cells migrated towards LPA at the concentration ranging from $0.1 \mathrm{nM}$ to $10 \mu \mathrm{M}$ (only the $1 \mu \mathrm{M}$ data is shown in Fig. 2a, an additional movie file shows this in more detail [see Additional file 5]).

BxPC3 cells were the most responsive to LPA of all the cell lines studied. Therefore, we quantitated the directionality and velocity of migration of BxPC3 cells in response to different concentrations of LPA. The directionality in response to LPA increased in a dose-dependent manner (Fig. $2 \mathrm{~b}$ left panel). The velocity also increased in a dosedependent manner in the dose range of 1 to $10 \mu \mathrm{M}$ LPA (Fig. 2b right panel). These results were in agreement the TAXIScan images (Fig. 2a). We confirmed the same phenomenon by an existing assay method, the Boyden chamber method. In the Boyden chamber method, BxPC3 cells showed good response to LPA in a dose-dependent manner (Fig. 2c, left). The concentrations of LPA that elicited the migration of $\mathrm{BxPC} 3$ cells were observed to be similar in both methods.

Expression of receptors for LPA on pancreatic cancer cells To confirm if the migration of cells was due to the LPAdependent phenomenon, we evaluated the expression of LPA receptors. Because most published reports showed either only mRNA expression or only protein expression $[12,13,27]$, we attempted to show both mRNA and protein expression systematically by using qRT-PCR and western blotting. As $\mathrm{LPA}_{1}, \mathrm{LPA}_{2}, \mathrm{LPA}_{3}, \mathrm{LPA}_{4}, \mathrm{LPA}_{5}$, and $\mathrm{LPA}_{6}$ are the known receptors for LPA; we used primers for these receptor isoforms (Additional file 1: Table S1) [27] to compare their mRNA expressions. In BxPC3 cells, based on the results of qRT-PCR, LPA 1 was the most highly expressed receptor among all the 6 receptors (Fig. 3a), whereas $\mathrm{LPA}_{2}, \mathrm{LPA}_{3}$, and $\mathrm{LPA}_{6}$ were moderately expressed and $\mathrm{LPA}_{5}$ showed the lowest expression. In PANC-1 cells, $\mathrm{LPA}_{1}$ and $\mathrm{LPA}_{3}$ were the major receptors expressed. In AsPC1 cells, the mRNA expression of $\mathrm{LPA}_{1}$, $\mathrm{LPA}_{2}$, and $\mathrm{LPA}_{6}$ were detected, and in MIAPaCa- 2 cells, the mRNA expression of most LPA receptors was extremely low. $\mathrm{LPA}_{3}$ expression was highest among the receptors for the MIAPaCa-2 cells (Fig. 3a).

We also evaluated the expression of these receptors at the protein level in the 4 pancreatic cell lines by western blotting using anti-LPA antibodies. All cell lines express a certain amount of $\mathrm{LPA}_{1}, \mathrm{LPA}_{2}$ and $\mathrm{LPA}_{3}$ receptors, however, very low expression of $\mathrm{LPA}_{4}, \mathrm{LPA}_{5}$, and $\mathrm{LPA}_{6}$ receptors was observed in lysates of all cell lines compared to $211 \mathrm{H}$, KATOIII or PC3 which were used as positive controls (Fig. 3b). The data from the migration assay and western blotting indicated that BxPC3 and PANC-1 cells express the LPA receptors and the migration images of the cells reflects the LPA-elicited migration.

\section{Signal transduction during migration of pancreatic cancer cells towards LPA}

To further confirm that the migration was LPA-dependent, we determined phosphorylation of various molecules in BxPC3 and PANC-1 cells using the PathScan array, which enabled us to simultaneously evaluate the phosphorylation of 39 different molecules (Additional file 2: Table S2). We carried out phosphorylation assays at the time points 0.5 , 2 , and 5 min following LPA stimulation, due to uniform stimulation of cells by LPA on culture dishes, which precludes the use of an LPA concentration gradient similar to that of the TAXI Scan device. Using this array system, we observed that Akt (Thr308 and Ser473), p44/42MAPK, IRS-1, InsR, c-kit, EphA2, and Tie2 were phosphorylated after LPA stimulation in both BxPC3 (Fig. 4a, b) and PANC-1 cells (Fig. 4c, d). Of these phosphorylated proteins, Akt and MAPK are known to be key molecules involved in migration and proliferation. The phosphorylation of these signaling molecules after uniform stimulation was further observed by western blotting. The results obtained showed that Akt (Thr308 and Ser473), p44/42MAPK were phosphorylated after LPA stimulation, as expected, in both BxPC3 and PANC-1 cell lines within 5 min (Figs. 4e and 5c). For the record, we also checked longer time points, such as $15,30,60,120$, and 240 min which were similar to the time points used in the TAXIScan experiments, but no additional increase in phosphorylation of these molecules 
a

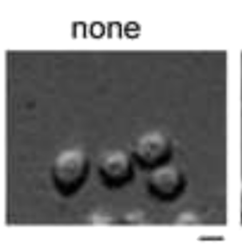

collagen I

fibronectin
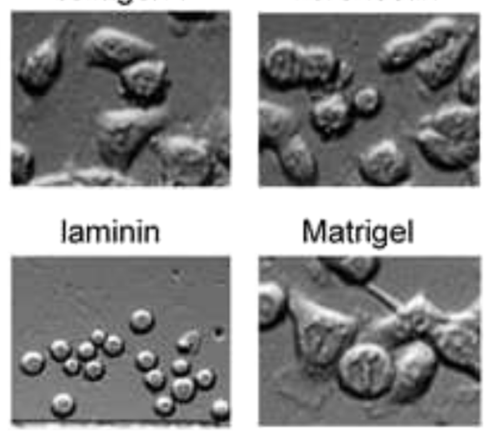

Matrigel

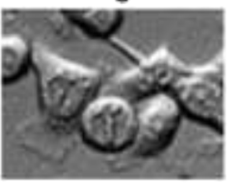

b

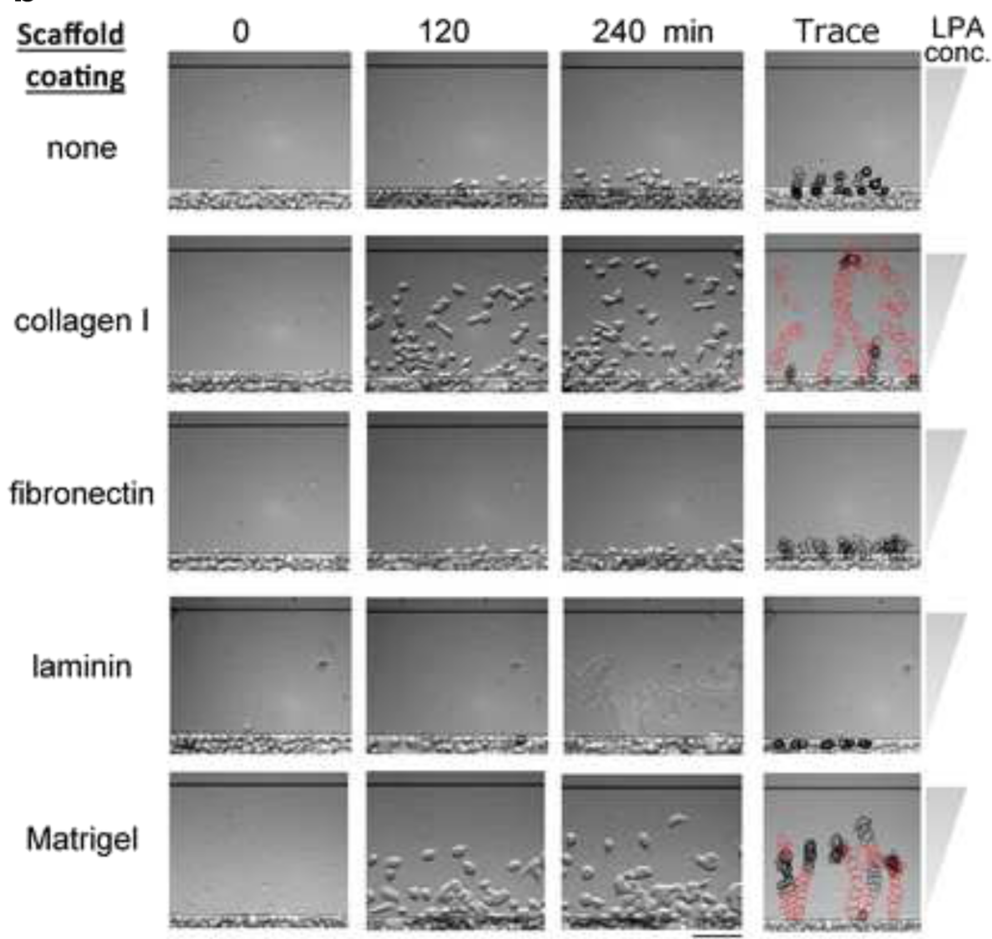

Fig. 1 Adhesion and migration of pancreatic cancer cells monitored by TAXIScan. a Morphology of BxPC3 pancreatic cancer cells after adherence to each scaffold material coated on the coverslip without chemoattractant. Images were taken 240 min after starting the assay. Scale bar: $10 \mu m$. b Chemotaxis of BXPC3 pancreatic cancer cells towards $100 \mathrm{nM}$ LPA with or without various kinds of scaffold-coating. Images taken at time 0, 120 and $240 \mathrm{~min}$ are shown. The morphologies of 4 or 5 representative migrating cells throughout the assays are shown on the "Trace" column. The outlines of the migrating cells were traced every $10 \mathrm{~min}$ in this column. Cells migrating at more than $1 \mu \mathrm{m} / \mathrm{min}$ are shown in red. All data are representative of 3 independent experiments. Scale bar: $100 \mu \mathrm{m}$

was observed (Fig. 4e). These data further support the establishment of the assay system of cancer cell migration towards LPA.

\section{Effect of inhibitor on migration towards LPA}

We also tested the effect of an LPA inhibitor, Ki16425 [28], on LPA-elicited migration of BxPC3 cells. When the cells were treated with Ki16425, the migration of the cells towards LPA was abrogated in a dose-dependent manner (Fig. 5a, b, an additional movie file shows this in more detail [see Additional file 6]). The half maximal inhibitory concentration $\left(\mathrm{IC}_{50}\right)$ value for directionality was $\approx 1.86 \mu \mathrm{M}$ (Fig. 5b, left graph). Owing to weak inhibition of velocity by Ki16425, the $\mathrm{IC}_{50}$ value for velocity was $>100 \mu \mathrm{M}$ (Fig. 5b, right graph). When the cells were treated $50 \mu \mathrm{M}$ Ki16425, the phosphorylation of Akt and MAPK was reduced, as observed during western blot analysis (Fig. 5c). The pancreatic cancer cells showed LPA-elicited chemotactic migration with clarity in the TAXIScan chamber, and this phenomenon was vigorously supported by the inhibition of the intracellular signaling with Ki16425. 

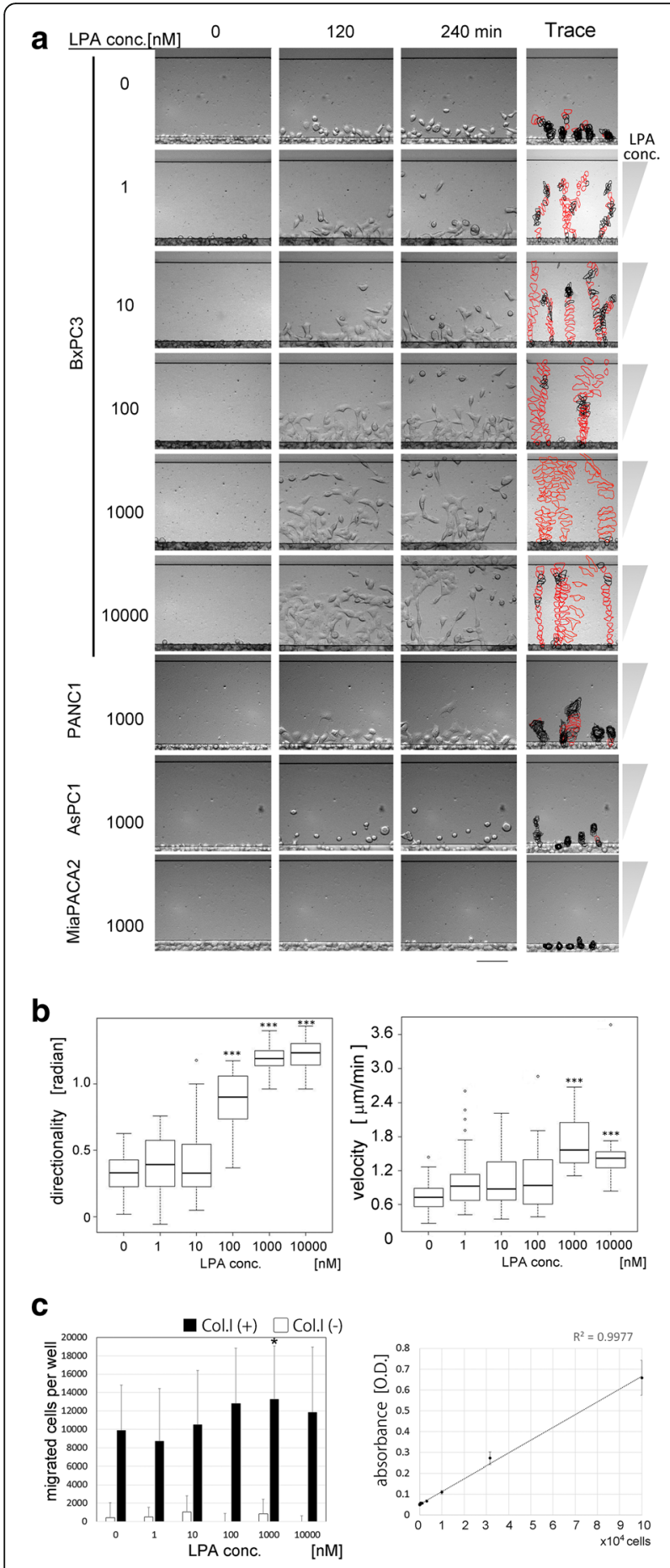

\section{Discussion}

In this study, we established a pancreatic cancer cell migration assay system by using the TAXIScan device. We found that coating of scaffolds such as collagen and Matrigel on glass, similar to that in some published studies using other methods, was necessary for successful adhesion and migration. BxPC3 and PANC-1 cells migrated towards LPA in a
Fig. 2 Chemotaxis of pancreatic cancer cells towards LPA detected by TAXIScan (a and $\mathbf{b}$ ) or Boyden chamber (c). a Four pancreatic cancer cell lines were used for the TAXIScan method. Dose-dependency of BxPC3 chemotaxis towards LPA is observed. The migration images of PANC-1, AsPC1, and MIAPaCa-2 cells in the optimal conditions are also shown. Images taken at time 0, 120 and $240 \mathrm{~min}$ are shown. The morphologies of 4 or 5 representative migrating cells throughout the assays are shown on the "Trace" column. The outlines of the migrating cells were recorded every $10 \mathrm{~min}$ in this column. Cells migrating at more than $1 \mu \mathrm{m} / \mathrm{min}$ are shown in red. Data are representative of 3 independent experiments. Scale bar: $100 \mu \mathrm{m}$. b Quantitation of the directionality and velocity of migration of $\mathrm{BxPC3}$ cells towards various concentrations of LPA. The graph on the left indicates the directionality and the graph on the right indicates velocity. White circles are outliers. Statistical analysis was done by the Kruskal-Wallis Test (Nonparametric ANOVA) followed by the Dunn's Multiple Comparisons Test. Data are representative of 3 independent experiments. c Migration of BxPC3 cells towards LPA using Boyden chamber assay kit. The migrated cells were stained with the staining solution and the numbers of the migrated cells were estimated by measuring OD $560 \mathrm{~nm}$ based on the standard curve (the graph on the right). The assay results with the collagen I coated membrane (black bar) or the plain membrane (white bar) are shown in the graph on the left. Mean values of data are shown and the error bars represents the standard error $(n=6)$. Statistical analysis was conducted using the Student's $t$-test. ${ }^{*} p<0.05$ (vs. data without LPA)

dose-dependent manner, which was clearly inhibited by an LPA inhibitor, Ki16425. This is the first report of pancreatic cancer cell migration monitored by the TAXIScan system that enables analysis of multiple parameters, including directionality, velocity, and cell morphology. Additionally, this is the first report simultaneously comparing the TAXIScan and Boyden chamber methods. The Boyden chamber method has been used for over 50 years [29], the limitations of this method have been pointed out by several researchers. In this method, a membrane of $10 \mu \mathrm{m}$ thickness, having holes of $8 \mu \mathrm{m}$ diameter (in this study) with random density, separates the upper and lower wells (see Additional file 7). It is thought that cells are able to sense differences in the chemoattractant concentration between these two wells. Although this method appears simple, it has certain limitations. (I) The density of holes may not be uniform. (II) The microstructure inside the hole, e.g., a micro-channel of $10 \mu \mathrm{m}$ length $\times 8 \mu \mathrm{m}$ diameter, is unknown, and the chemoattractant gradient is not measurable. (III) A large number of cells is necessary for this assay $\left(1.5 \times 10^{5}\right.$ cells per well in this study). (IV) A considerable amount of chemoattractant is necessary (500 $\mu \mathrm{L}$ per well in this study), which is expensive. (V) The process of cell migration is not visible. (VI) The device only displays the numbers of migrated cells. (VII) The obtained data may have high background noise. (VIII) The density of cells migrating to the lower side of the membrane is not uniform. A few advantage of this method are as follows: (I) It has a simple structure; (II) the apparatus itself (without coating 
a

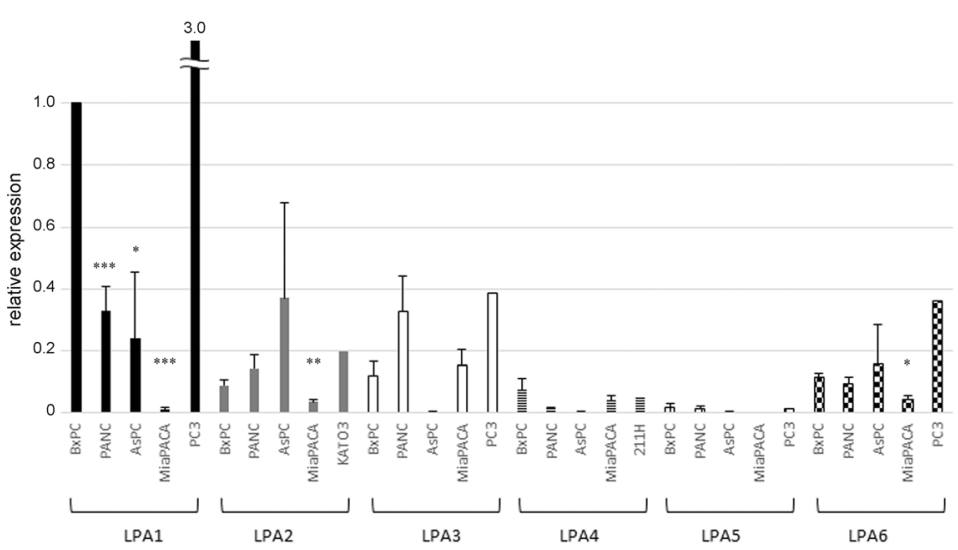

b
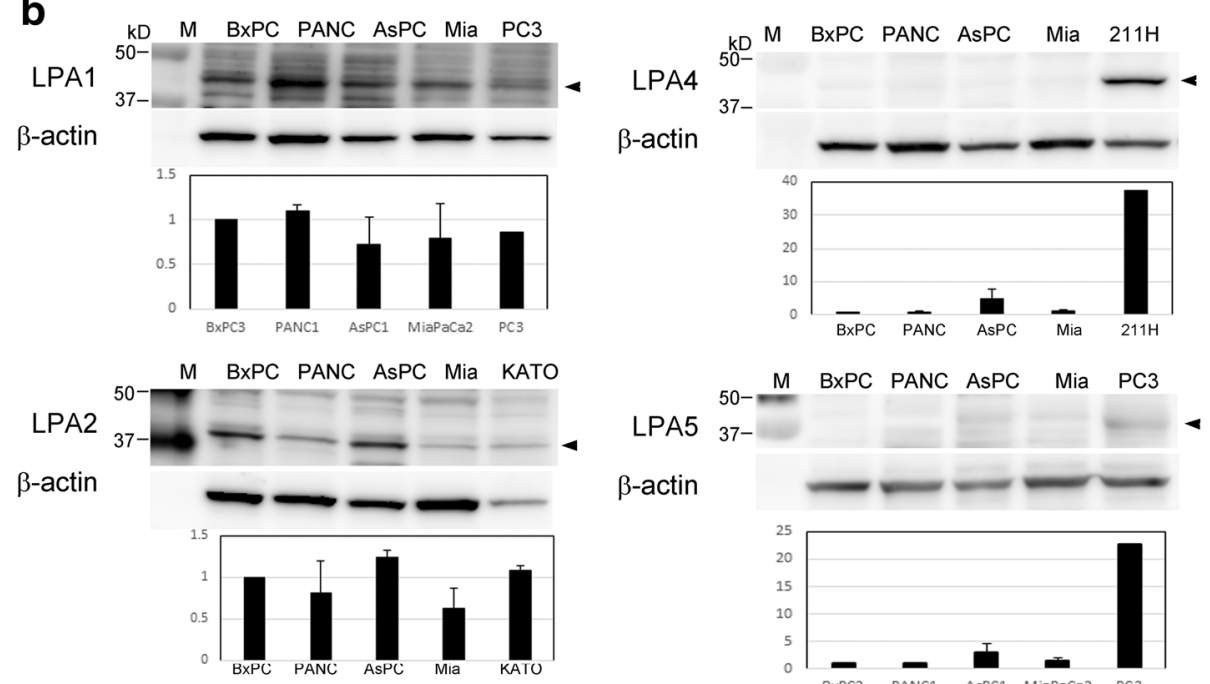

M BXPC PANC AsPC Mia PC3
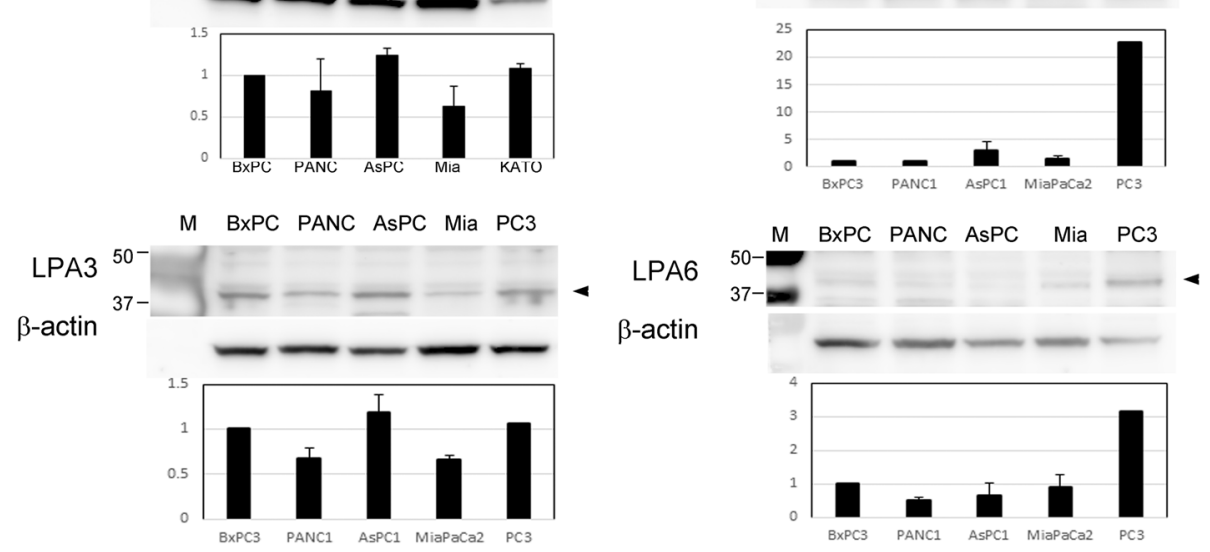

Fig. 3 LPA receptor expression in pancreatic cancer cell lines. a mRNA expression in 4 pancreatic cancer cell lines determined by quantitative RT-PCR. The relative expression of each receptor was calculated based on the LPA 1 expression in BxPC3. Data represent mean values of 3 independent experiments. The error bars represent the standard error. Statistical analysis was conducted using the Student's $t$-test. ${ }^{*} p<0.05,{ }^{* *} p<0.01$,

${ }^{* * *} p<0.001$ (vs. BxPC3). b Protein expression in 4 pancreatic cancer cell lines detected by SDS-PAGE and western blotting. A prostate cancer cell line, PC3, a gastric cancer cell line, KATOIII, and a pleural mesothelioma cell line, $211 \mathrm{H}$, were used as positive controls. $\beta$-actin was used as a loading control and its expression is also shown. The arrow-head indicates the specific bands of each LPA receptor. M, protein marker; Mia, MIAPaCa-2; Photographs are representative of 3 independent experiments. The intensity of each band was measured and the relative expression of each receptor protein was calculated based on the receptor in BXPC3 cells. Quantitative data represent mean values of 3 independent experiments except the positive controls PC3 and $211 \mathrm{H}$. The error bars represent the standard error

materials) is inexpensive; and (III) it is well known and widely used. On the other hands, the advantages of TAXIScan are as follows [14] (see also Additional file 8): (I) it has an uniform micro-channel $(260 \mu \mathrm{m}$ length $\times 1000 \mu \mathrm{m}$ width $\times 8 \mu \mathrm{m}$ height); (II) the chemoattractant, which is placed on one end of the micro-channel, defuses uniformly through the channel, resulting in a stable concentration gradient [14]; (III) a small number of cells 


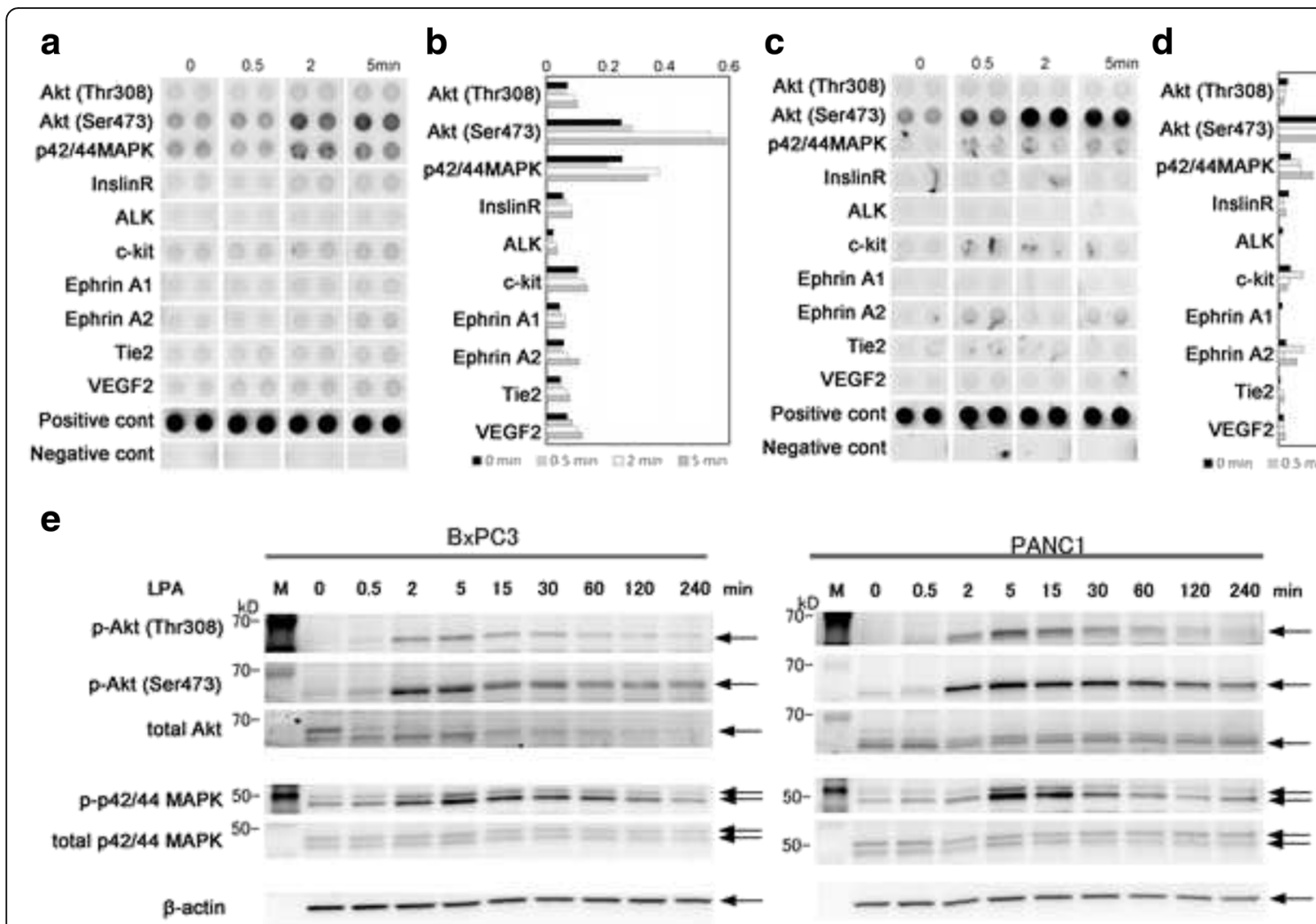

Fig. 4 Phosphorylation of receptors or signaling molecules. a and $\mathbf{c}$ Images of phosphorylation of receptors in BxPC3 (a) or in PANC-1 (c) cell lines detected by Antibody Array. Data are representative of 3 independent experiments. $\mathbf{b}$ and $\mathbf{d}$ The quantitation of phosphorylation by measuring density of Antibody Array with BxPC3 data (b) or with PANC-1 data (d). e Phosphorylation of Akt or p44/42MAPK in BxPC3 and PANC-1 cell lines, as indicated. Cell lysates taken after LPA stimulation at each time point were analyzed by SDS-PAGE and western blotting. Anti- $\beta$-actin antibody was used as the internal control. Arrows indicate the specific band for each antibody. Data are representative of 2 independent experiments

is required for analysis (100 or less cells per channel); (IV) a small and inexpensive amount of chemoattractant is necessary (1 $\mu \mathrm{L}$ per channel); (V) migrating cells are observable; (VI) images obtained during migration are recorded automatically; (VII) data obtained from this assay including that on morphology, behavior, directionality, and velocity, are more informative. However, some demerits of TAXIScan are as follows: (I) although the running cost is low, the initial cost is high, and (II) it is not well-known yet. In fact, it may not be appropriate to position TAXIScan as an alternate to the Boyden method, because both methods utilize completely different equipment and data collection methods, and the quality of data obtained using these methods is entirely different (Additional files 7 and 8). However, because of lower requirement of samples and the collection of more informative data, the approach to cancer cell migration using TAXIScan is more useful than analysis using existing techniques such as the Boyden chamber method. With the TAXIScan system, the characteristics of pancreatic cancer cells can be analyzed in detail. Moreover, our system can be adopted for migration studies in other types of cancer cells.
In the Boyden chamber method, a certain number of cells without LPA was observed to migrate, indicating a high background (Fig. 2c), similar to that reported previously [30-33]. This high background with the Boyden chamber method is considered to be due to the thickness of the membrane $(10 \mu \mathrm{m}$ in this study). In TAXIScan method, cells without LPA were observed to migrate for more than $10 \mu \mathrm{m}$ (up to $100 \mu \mathrm{m}$ ) (Fig. 2a), explaining this phenomenon. From this point of view, we could argue that TAXIScan has a wider dynamic range to detect cell migration.

Herein, 4 pancreatic cancer cell lines were analyzed and only 2 of these cell-lines, BxPC3 and PANC-1, showed good migration towards LPA with reasonable co-evidence on the expression of LPA receptors. The reason why AsPC1 and $\mathrm{MIAPaCa}-2$ cells do not migrate towards LPA is still unknown. BxPC3 and PANC-1 do express $\mathrm{LPA}_{1}, \mathrm{LPA}_{2}$, and $\mathrm{LPA}_{3}$; however, these cell lines do not express $\mathrm{LPA}_{4}, \mathrm{LPA}_{5}$, and $\mathrm{LPA}_{6}$ as observed during western blotting (Fig. 3b). The latter 3 receptors are likely not involved in cell migration but might be involved in other cellular functions. 


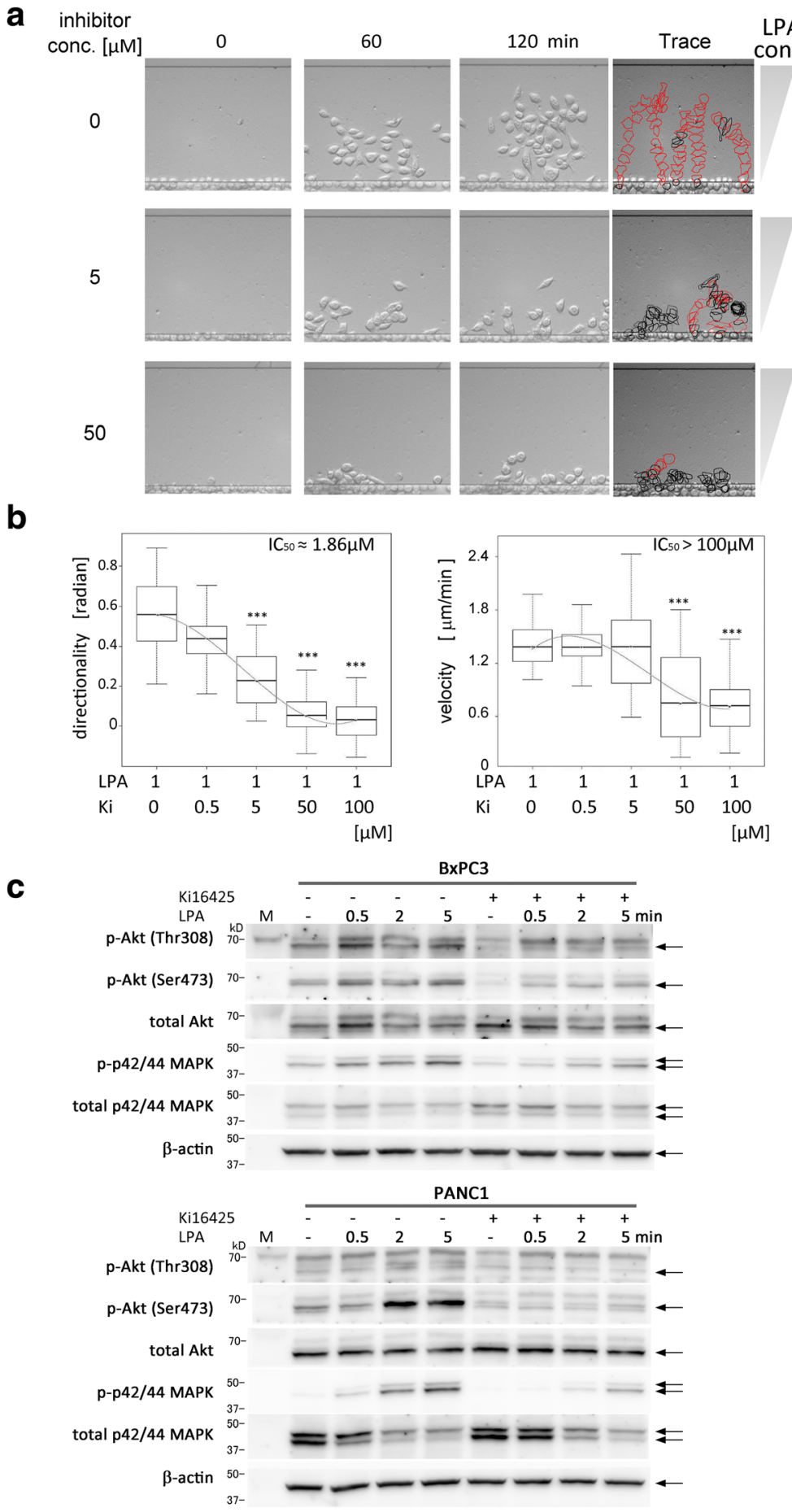

Fig. 5 Inhibition of BxPC3 chemotaxis towards LPA by Ki16425. a BxPC3 chemotaxis towards 1 MM LPA with various concentrations of Ki16425. Cells were pre-incubated with Ki16425 for $24 \mathrm{~h}$ and the chemotaxis assay was performed using TAXIScan. Data are representative of 3 independent experiments. $\mathbf{b}$ Box-plots of the directionality and the velocity in BXPC3 migration towards LPA with Ki16425. The graph on the left indicates directionality and that on the right indicates velocity. The half maximal inhibitory concentration $\left(I_{50}\right)$ values are also shown. Statistical analysis was done by the Kruskal-Wallis Test (Non-parametric ANOVA) followed by the Dunn's Multiple Comparisons Test. ${ }^{* *} p<0.0001$ (vs. data with $1 \mu \mathrm{M}$ LPA and without Ki16425). Data are representative of 3 independent experiments. c Inhibition of phosphorylation of Akt or p44/42MAPK by Ki16425 in BXPC3 and PANC-1 cell lines, as indicated. Cell lysates taken after LPA stimulation at each time point were analyzed by SDS-PAGE and western blotting. Anti- $\beta$-actin antibody was used as the internal control. Arrows indicate the specific band for each antibody. Data are representative of 3 independent experiments 
LPA inhibitor, Ki16425, shown in this study is believed to block human $\mathrm{LPA}_{1}$ and $\mathrm{LPA}_{3}$ receptors [28]; $10 \mu \mathrm{M}$ of Ki16425 significantly blocked the migration of cancer cells [13]. In our system, Ki16425 clearly inhibited BxPC3 cell migration towards LPA at 5-50 $\mu \mathrm{M}$ concentrations, indicating that TAXIScan and BxPC3 cells are the best tools for screening inhibitors of pancreatic cell migration. Utilizing such a new method, new molecules for regulating pancreatic cancer metastasis can be identified, and the limited treatment options and the poor prognosis of this disease can be overcome. Studies on neutrophils have tested various kinds of compounds and found that some compounds inhibit neutrophil function, leading to the successful selection of several effective molecules [34]. Collectively, it can be concluded that the system established in our study can be a powerful tool for cancer research and drug discovery in seeking effectors and inhibitors for analyzing cancer cell function. We are currently looking for and screening such molecules that can regulate pancreatic cancer cell migration; some promising molecules will be reported in the near future.

\section{Conclusions}

We established a novel pancreatic cancer cell migration assay system that provides optical and quantitative information simultaneously. Using this system, we demonstrated that BxPC3 and PANC-1 cells showed good migration towards LPA. The effect of an LPA inhibitor, Ki16425, was detected clearly in this system, which was confirmed by the reduction in the phosphorylation of signal transduction molecules, Akt and MAPK. As this method provides a large amount of information on migrating cells simultaneously, such as their morphology, directionality, and velocity, with a small volume of sample, it can be a powerful tool for analyzing the characteristics of cancer cells and for evaluating factors affecting cellular functions.

\section{Additional files}

Additional file 1: Table S1. Primers used for the quantitative RT-PCR. Total 6 pairs of primers for LPA receptors (LPA1, LPA2, LPA3, LPA4, LPA 5, and LPA6) were used for this study, based on the information reported. previously (27). (DOCX $14 \mathrm{~kb}$ )

Additional file 2: Table S2. Targets for PathScan RTK signaling array. The phosphorylation of 39 different molecules in BxPC3 and PANC-1 cells was evaluated using the PathScan array. Details are described in Methods section. (DOCX $14 \mathrm{~kb}$ )

Additional file 3: Chemotaxis of BXPC3 pancreatic cancer cells towards $100 \mathrm{nM}$ LPA with or without various kinds of scaffold-coating. Images were taken every $5 \mathrm{~min}$ for $4 \mathrm{~h}$ and movies were created by TAXIScan Analyzer2 software. Representative of 3 independent experiments. Scale bar: $100 \mu \mathrm{m}$. (MP4 $9312 \mathrm{~kb})$

Additional file 4: Chemotaxis of BXPC3 pancreatic cancer cells towards various concentrations of LPA on a collagen I coated coverslip. Images were taken every $5 \mathrm{~min}$ for $4 \mathrm{~h}$ and movies were created by TAXIScan Analyzer2 software. Representative of 3 independent experiments. Scale bar: $100 \mu \mathrm{m}$. (MP4 $7612 \mathrm{~kb})$

Additional file 5: Chemotaxis of four kinds of pancreatic cancer cells towards $1 \mu \mathrm{M}$ LPA on a collagen I coated coverslip. Images were taken every $5 \mathrm{~min}$ for $4 \mathrm{~h}$ and movies were created by TAXIScan Analyzer2 software. Representative of 3 independent experiments. Scale bar: $100 \mu \mathrm{m}$. (MP4 $6258 \mathrm{~kb})$

Additional file 6: Inhibition of BxPC3 chemotaxis towards LPA by Ki16425. Cells were pre-incubated with Ki16425 for $24 \mathrm{~h}$ and the chemotaxis assay towards $1 \mu \mathrm{M}$ LPA was performed using TAXIScan. Images were taken every $5 \mathrm{~min}$ for $4 \mathrm{~h}$ and movies were created by TAXIScan Analyzer2 software. Representative of 3 independent experiments. Scale bar: $100 \mu \mathrm{m}$. (MP4 6072 kb)

Additional file 7: The modified Boyden chamber assay. A) Schematic diagram (sagittal section) of one well of the modified Boyden chamber assay (Transwell). Cells in the chemotaxis buffer are located in the upper chamber and the chemoattractant the chemotaxis buffer is added to the lower chamber. B) Schematic diagram of the membrane part of the modified Boyden chamber. The membrane separates the upper and the lower chamber. The matrix is coated on the lower side of the membrane. C) Photographs of the lower side of the membrane after the assay. Cells are stained with the staining solution accompanied with the assay kit. Magnification: 400x. (TIFF $98113 \mathrm{~kb}$ )

Additional file 8: The TAXIScan assay. A) Schematic diagram (sagittal section) of one channel of the TAXIScan chamber. The chamber is filled with the chemotaxis buffer (light brown color). Cells are located on the one side of the micro-channel and the chemoattractant (red color) is placed on the other side of the micro-channel. B) Schematic diagram (sagittal section) of the micro-channel. The chemoattractant is defused in the micro-channel, which forms the stable concentration gradient. Cells on the matrix-coated coverslip migrates towards the gradient of the chemoattractant in the micro-channel. C) Photograph of cells migrating towards the chemoattractant. The image is taken from underneath of the TAXIScan chamber. (TIFF $98112 \mathrm{~kb}$ )

\section{Abbreviations}

BSA: Bovine serum albumin; EDTA: Ethylenediamine tetraacetic acid; EGF: Epidermal growth factor; Eph: Ephrin; FBS: Fetal bovine serum; IC50: The half maximal inhibitory concentration; InsR: Insulin receptor; IRS-1: Insulin receptor substrate 1; LPA: Lysophosphatidic acid; MAPK: Mitogen-activated protein kinase; RTK: Receptor tyrosine kinase; SDS-PAGE: Sodium dodecyl sulfate-polyacrylamide gel electrophoresis; Tie2: Tyrosine kinase with Ig-like loops and epidermal growth factor homology domains-2

\section{Acknowledgements}

We would like to thank Dr. Masakiyo Sakaguchi for providing materials, Editage (www.editage.jp) for English language editing, and the central research center of Kawasaki Medical School for technical supports.

\section{Funding}

This study was supported by JSPS KAKENHI Grant Number JP15K10201 (to AY), JP25861742 / JP16K11470 (to NK), and JP15K09671 (to FK), Wesco Scientific Promotion Foundation (to AY), Kawasaki Medical foundation for Medicine and Medical Welfare (to AY), and Kawasaki Medical School projectresearch fund (to AY, MY, and NK). There was no role with all funding bodies above in the design of the study or collection, analysis, or interpretation of the data or writing the manuscript.

\section{Availability of data and materials}

All data and materials are available upon reasonable request to the corresponding author. The data in this study were not deposited in publicly available repositories since there is no suitable repository service for the data.

\section{Authors' contributions}

AY and MY overviewed and designed this study and analyzed data. NK, MI, $\mathrm{KK}$, and NO collected and analyzed data. MN, YY, and FK critically discussed and corrected the manuscript. All authors have read and approved the manuscript. 


\section{Competing interests}

There is no competing interest regarding the publication of this paper.

\section{Consent for publication}

Not applicable since no personal information was collected in this study.

\section{Ethics approval and consent to participate}

Not applicable since the established cell lines used in this study had no personal information.

\section{Publisher's Note}

Springer Nature remains neutral with regard to jurisdictional claims in published maps and institutional affiliations.

\section{Author details \\ 'Department of Biochemistry, Kawasaki Medical School, 577 Matsushima, Kurashiki, Okayama 701-0192, Japan. Department of Clinical Oncology, Kawasaki Medical School, Okayama, Japan. ${ }^{3}$ Department of Molecular and Developmental Biology, Kawasaki Medical School, Okayama, Japan. ${ }^{4}$ Department of Surgery and Oncology, Graduate School of Medical Sciences, Kyushu University, Fukuoka, Japan.}

\section{Received: 14 March 2016 Accepted: 22 March 2017}

Published online: 31 March 2017

\section{References}

1. Roussos ET, Condeelis JS, Patsialou A. Chemotaxis in cancer. Nat Rev Cancer. 2011:11(8):573-87.

2. Dorsam RT, Gutkind JS. G-protein-coupled receptors and cancer. Nat Rev Cancer. 2007;7(2):79-94.

3. Murph M, Tanaka T, Liu S, Mills GB. Of spiders and crabs: the emergence of lysophospholipids and their metabolic pathways as targets for therapy in cancer. Clin Cancer Res. 2006;12(22):6598-602.

4. Hecht JH, Weiner JA, Post SR, Chun J. Ventricular zone gene-1 (vzg-1) encodes a lysophosphatidic acid receptor expressed in neurogenic regions of the developing cerebral cortex. J Cell Biol. 1996;135(4):1071-83.

5. An S, Bleu T, Hallmark OG, Goetzl EJ. Characterization of a novel subtype of human $\mathrm{G}$ protein-coupled receptor for lysophosphatidic acid. J Biol Chem. 1998;273(14):7906-10.

6. Bandoh K, Aoki J, Hosono H, Kobayashi S, Kobayashi T, Murakami-Murofushi K, Tsujimoto M, Arai H, Inoue K. Molecular cloning and characterization of a novel human G-protein-coupled receptor, EDG7, for lysophosphatidic acid. J Biol Chem. 1999;274(39):27776-85.

7. Noguchi K, Ishii S, Shimizu T. Identification of p2y9/GPR23 as a novel G protein-coupled receptor for lysophosphatidic acid, structurally distant from the Edg family. J Biol Chem. 2003;278(28):25600-6.

8. Kotarsky K, Boketoft A, Bristulf J, Nilsson NE, Norberg A, Hansson S, Owman C, Sillard R, Leeb-Lundberg LM, Olde B. Lysophosphatidic acid binds to and activates GPR92, a G protein-coupled receptor highly expressed in gastrointestinal lymphocytes. J Pharmacol Exp Ther. 2006;318(2):619-28.

9. Pasternack SM, von Kügelgen I, Al Aboud K, Lee YA, Rüschendorf F, Voss K, Hillmer AM, Molderings GJ, Franz T, Ramirez A, et al. G protein-coupled receptor P2Y5 and its ligand LPA are involved in maintenance of human hair growth. Nat Genet. 2008;40(3):329-34.

10. Chun J, Hla T, Lynch KR, Spiegel S, Moolenaar WH. International Union of Basic and Clinical Pharmacology. LXXVIII. Lysophospholipid receptor nomenclature. Pharmacol Rev. 2010;62(4):579-87.

11. Mills GB, Moolenaar WH. The emerging role of lysophosphatidic acid in cancer. Nat Rev Cancer. 2003;3(8):582-91.

12. Stähle M, Veit C, Bachfischer U, Schierling K, Skripczynski B, Hall A, Gierschik $P$, Giehl K. Mechanisms in LPA-induced tumor cell migration: critical role of phosphorylated ERK. J Cell Sci. 2003;116(Pt 18):3835-46.

13. Yamada T, Sato K, Komachi M, Malchinkhuu E, Tobo M, Kimura T, Kuwabara A, Yanagita Y, Ikeya T, Tanahashi Y, et al. Lysophosphatidic acid (LPA) in malignant ascites stimulates motility of human pancreatic cancer cells through LPA1. J Biol Chem. 2004;279(8):6595-605.

14. Kanegasaki S, Nomura Y, Nitta N, Akiyama S, Tamatani T, Goshoh Y, Yoshida T, Sato T, Kikuchi Y. A novel optical assay system for the quantitative measurement of chemotaxis. J Immunol Methods. 2003;282(1-2):1-11.

15. Jang MH, Sougawa N, Tanaka T, Hirata T, Hiroi T, Tohya K, Guo Z, Umemoto E, Ebisuno Y, Yang BG, et al. CCR7 is critically important for migration of dendritic cells in intestinal lamina propria to mesenteric lymph nodes. J Immunol. 2006:176(2):803-10.

16. Nishio M, Watanabe K, Sasaki J, Taya C, Takasuga S, lizuka R, Balla T,

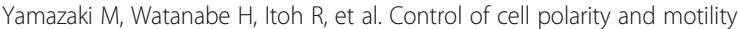
by the Ptdlns(3,4,5)P3 phosphatase SHIP1. Nat Cell Biol. 2007;9(1):36-44.

17. Ito A, Suganami T, Yamauchi A, Degawa-Yamauchi M, Tanaka M, Kouyama R, Kobayashi Y, Nitta N, Yasuda K, Hirata Y, et al. Role of CC Chemokine receptor 2 in bone marrow cells in the recruitment of macrophages into obese adipose tissue. J Biol Chem. 2008;283(51):35715-23.

18. Nishikimi A, Fukuhara H, Su W, Hongu T, Takasuga S, Mihara H, Cao Q, Sanematsu F, Kanai M, Hasegawa $H$, et al. Sequential regulation of DOCK2 dynamics by two phospholipids during neutrophil chemotaxis. Science. 2009;324(5925):384-7.

19. Uchida M, Oyanagi E, Miyachi M, Yamauchi A, Yano H. Relationship between macrophage differentiation and the chemotactic activity toward damaged myoblast cells. J Immunol Methods. 2013;393(1-2):61-9.

20. Yamauchi A, Degawa-Yamauchi M, Kuribayashi F, Kanegasaki S, Tsuchiya T. Systematic single cell analysis of migration and morphological changes of human neutrophils over stimulus concentration gradients. J Immunol Methods. 2014;404:59-70.

21. Kurose $K$, Ohue Y, Sato E, Yamauchi A, Eikawa S, Isobe M, Nishio Y, Uenaka A, Oka M, Nakayama E. Increase in activated Treg in TIL in lung cancer and in vitro depletion of Treg by ADCC using an antihuman CCR4 mAb (KM2760). J Thorac Oncol. 2015;10(1):74-83.

22. Ishii M, Egen JG, Klauschen F, Meier-Schellersheim M, Saeki Y, Vacher J, Proia RL, Germain RN. Sphingosine-1-phosphate mobilizes osteoclast precursors and regulates bone homeostasis. Nature. 2009;458(7237):524-8.

23. Nitta N, Tsuchiya T, Yamauchi A, Tamatani T, Kanegasaki S. Quantitative analysis of eosinophil chemotaxis tracked using a novel optical device - TAXIScan. J Immunol Methods. 2007;320(1-2):155-63.

24. Terashima Y, Onai N, Murai M, Enomoto M, Poonpiriya V, Hamada T, Motomura K, Suwa M, Ezaki T, Haga T, et al. Pivotal function for cytoplasmic protein FROUNT in CCR2-mediated monocyte chemotaxis. Nat Immunol. 2005;6(8):827-35.

25. Takamatsu H, Takegahara N, Nakagawa Y, Tomura M, Taniguchi M, Friedel RH, Rayburn H, Tessier-Lavigne M, Yoshida Y, Okuno T, et al. Semaphorins guide the entry of dendritic cells into the lymphatics by activating myosin II. Nat Immunol. 2010;11(7):594-600.

26. Yamauchi A, Hadjur C, Takahashi T, Suzuki I, Hirose K, Mahe YF. Human skin melanocyte migration towards stromal cell-derived factor-1 alpha demonstrated by optical real-time cell mobility assay: modulation of their chemotactic ability by alpha-melanocyte-stimulating hormone. Exp Dermatol. 2013;22(10):664-7.

27. Jongsma M, Matas-Rico E, Rzadkowski A, Jalink K, Moolenaar WH. LPA is a chemorepellent for B16 melanoma cells: action through the CAMP-elevating LPA5 receptor. PLoS One. 2011;6(12):e29260.

28. Ohta H, Sato K, Murata N, Damirin A, Malchinkhuu E, Kon J, Kimura T, Tobo M, Yamazaki Y, Watanabe T, et al. Ki16425, a subtype-selective antagonist for EDGfamily lysophosphatidic acid receptors. Mol Pharmacol. 2003;64(4):994-1005.

29. BOYDEN S. The chemotactic effect of mixtures of antibody and antigen on polymorphonuclear leucocytes. J Exp Med. 1962;115:453-66.

30. Schuller HM, Al-Wadei HA, Majidi M. GABA B receptor is a novel drug target for pancreatic cancer. Cancer. 2008;112(4):767-78.

31. Schuller HM, Al-Wadei HA, Majidi M. Gamma-aminobutyric acid, a potential tumor suppressor for small airway-derived lung adenocarcinoma. Carcinogenesis. 2008;29(10):1979-85

32. König J, Weiss G, Rossi D, Wankhammer K, Reinisch A, Kinzer M, Huppertz B, Pfeiffer D, Parolini O, Lang I. Placental mesenchymal stromal cells derived from blood vessels or avascular tissues: what is the better choice to support endothelial cell function? Stem Cells Dev. 2015;24(1):115-31.

33. Kestens C, Siersema PD, Offerhaus GJ, van Baal JW. BMP4 signaling is able to induce an epithelial-Mesenchymal transition-like phenotype in Barrett's esophagus and esophageal Adenocarcinoma through induction of SNAIL2. PLoS One. 2016;11(5):e0155754.

34. Hattori H, Subramanian KK, Sakai J, Jia Y, Li Y, Porter TF, Loison F, Sarraj B, Kasorn A, Jo H, et al. Small-molecule screen identifies reactive oxygen species as key regulators of neutrophil chemotaxis. Proc Natl Acad Sci U S A. 2010;107(8):3546-51. 